\title{
Combining Ability Studies for Yield, Yield Components and Nutritional Traits in Greengram (Vigna radiata (L.) Wilczek)
}

\author{
S. Kalpana*, N.V. Naidu and D.M. Reddy \\ Department of Genetics and Plant Breeding, S.V. Agricultural College, Tirupati - 517502, \\ A.P., India \\ *Corresponding author
}

\section{A B S T R A C T}

\section{Keywords}

Combining ability, Mungbean, gca, sca, Yield components

Article Info

Accepted:

15 October 2018 Available Online:

10 November 2018
Five lines were crossed with four testers in $\mathrm{L} x \mathrm{~T}$ fashion to assess the general combining ability of parents, specific combining ability of crosses and to determine the mode of gene action involved in the inheritance of yield attributes and nutritional traits. The analysis of variance for combining ability revealed higher magnitude of sca variances than gca variances denotes the predominance of non-additive gene action for most of the yield contributing traits and nutritional traits. Further the ratio of variance due to general and specific combining ability was less than unity for all the traits also confirmed the role of non-additive gene action in governing these traits. LGG-407, LGG-460, LGG-574, Pusa Vishal, IPM-2-14, and PM-5 were identified as best combiners for most of the yield and nutritional traits. Based on the per se performance and sca effects the crosses LGG-574 $\times$ Pusa Vishal, LGG-574 × PM-5, LGG-460 × Pusa Vishal, LGG-460 × IPM-2-14 and LGG$407 \times$ PM-5 were identified as superior crosses that could be exploited for developing high yielding lines with improved nutritional traits in greengram.

\section{Introduction}

Greengram is third most important and highly valued legume crop in India after chickpea and pigeon pea. It is an outstanding source of palatable, nutritive, easily digestible, high quality non-flatulent proteins than other pulses and constitutes an important source of cereal based diet in Asia (Kamleswar et al., 2014). The low productivity in greengram is due its cultivation under rainfed situation on marginal lands with low input application and also use of low yielding cultivars (Reddy et al., 2011). The genetic potential of present day cultivars of mungbean can be improved by employing diverse paents in hybridization programme. The combining ability analysis serves as an efficient tool for selection of desirable parents for hybridization and also aids in screening of promising crosses. General combining ability variance is mainly attributed to additive $x$ additive interactions, whereas specific combining ability variance is a consequence of dominance $\times$ dominance epistatic interactions.

The high yielding lines may not necessarily be able to transmit their superiority to their hybrids (Allard, 1960). Therefore the estimates of gca and sca may be of more reliable rather than per se performance of 
genotypes. Hence an attempt has been made to estimate $g c a$ and sca gene effects for yield components and nutritional traits in greengram through line $\times$ tester mating design.

\section{Materials and Methods}

Five lines viz., TM-96-2, MGG-295, LGG574, LGG-460 and LGG-407 were crossed with each of four testers viz., Pusa Vishal, PM-5, IPM-2-14 and PM-110 in a Line $x$ Tester mating design and $20 \mathrm{~F}_{1}$ swere produced during kharif, 2016. The 20 crosses along with nine parents were grown in Randomised Complete Block Design (RCBD) with three replications during rabi, 2016-17 at S.V. Agricultural College Farm, Tirupati. Each entry in each replication was grown in two rows of $3 \mathrm{~m}$ length. The spacing adopted between the rows was $30 \mathrm{~cm}$ and within a row between the plants was $10 \mathrm{~cm}$. All recommended crop production and protection practices were followed to raise a good and healthy crop. Data was recorded on five randomly selected plants in each genotype in each replication. Mean values on plant basis were recorded for traits like plant height, number of branches per plant, number of clusters per plant, number of pods per cluster, 100 seed weight, seed yield per plant while the traits days to $50 \%$ flowering and days to maturity were recorded on plot basis. The mean performance of parents and crosses is represented in table 2. Mean data of all the traits was subjected to analysis of variance as per Panse and Sukhatme (1985) to test the significance levels. Linex tester analysis was carried out as given by Kempthorne (1957).

\section{Results and Discussion}

Analysis of variance for combining ability (Table 2) revealed the presence of significant variability for all the traits under study for parents, whereas crosses had significant variability for all traits except days to maturity. Significance of mean sum of squares due to parents $v s$ crosses for all traits except days to maturity, number of branches per plant, reducing sugars indicated the presence of substantial variability in crosses for the traits. Mean sum of squares due to lines were found to be significant for all the characters except number of seeds per pod revealing the major contribution of lines towards components of general combining ability variance for most of the characters. Mean sum of squares due to testers were also significant for all the traits except days to 50 percent flowering, number of branches per plant, number of clusters per plant suggesting the significant contribution of testers towards general combining ability variance components. Mean sum of squares due to line $x$ tester interaction effects were also found to be significant for 12 characters except for the days to 50 percent flowering, and days to maturity indicating the significant contribution of crosses towards components of specific combining ability variance. The ratio of $g c a$ variance to $s c a$ variance ranged from 0.002 to 0.461 indicated the preponderance of nonadditive gene action for majority of yield components and nutritional traits.

The appropriate choice of parents for hybridization predominantly determines the success of any breeding programme. The knowledge of general combining ability coupled with high per se performance would help in selection of potential parents with superior genes (Singh and Harisingh, 1985). The estimates of $\mathrm{gca}$ of parents were presented in table 3. Based on per se performance and gca effects, LGG-407 was identifies as best parents for., number of branches per plant, number of clusters per plant, number of seeds per pod, seed yield per plant, total protein content, total sugars and non-reducing sugars. LGG-574 was found to good parent for 100 seed weight, seed yield per plant, harvest index and total sugars (Table $1)$. 


\section{Int.J.Curr.Microbiol.App.Sci (2018) 7(11): 1771-1779}

Table.1 Mean performance of parents and crosses for seed yield, yield components and nutritional quality traits in greengram

\begin{tabular}{|c|c|c|c|c|c|c|c|c|c|c|c|c|c|c|}
\hline Genotype & $\begin{array}{c}\text { Days to } \\
\mathbf{5 0 \%} \\
\text { flowering }\end{array}$ & $\begin{array}{l}\text { Days to } \\
\text { maturity }\end{array}$ & $\begin{array}{l}\text { Plant } \\
\text { height } \\
\text { (cm) }\end{array}$ & $\begin{array}{c}\text { Number } \\
\text { of } \\
\text { branches }^{-1} \\
\text { plant }^{-1}\end{array}$ & $\begin{array}{c}\text { Number } \\
\text { of } \\
\text { clusters }^{-} \\
\text {plant }^{-1}\end{array}$ & $\begin{array}{l}\text { Number } \\
\text { of pods } \\
\text { cluster }^{-1}\end{array}$ & $\begin{array}{c}\text { Number } \\
\text { of seeds } \\
\text { pod }^{-1}\end{array}$ & $\begin{array}{c}100 \\
\text { seed } \\
\text { weight } \\
(\mathrm{g})\end{array}$ & $\begin{array}{c}\text { Seed } \\
\text { yield } \\
\text { plant } \\
{ }^{1}(g)\end{array}$ & $\begin{array}{c}\text { Harvest } \\
\text { index } \\
(\%)\end{array}$ & $\begin{array}{c}\text { Total } \\
\text { protein } \\
\text { content } \\
(\%)\end{array}$ & $\begin{array}{c}\text { Total } \\
\text { sugars } \\
(\%)\end{array}$ & $\begin{array}{c}\text { Reducing } \\
\text { sugars } \\
(\%)\end{array}$ & $\begin{array}{l}\text { Non- } \\
\text { reducing } \\
\text { sugars } \\
(\%)\end{array}$ \\
\hline \multicolumn{15}{|l|}{ Lines } \\
\hline TM-96-2 & 39.67 & 71.00 & 35.56 & 1.00 & 3.90 & 2.97 & 10.49 & 3.57 & 9.72 & 36.35 & 24.14 & 7.69 & 0.68 & 7.01 \\
\hline MGG-295 & 36.33 & 67.33 & 39.98 & 2.00 & 4.16 & 2.96 & 10.11 & 3.85 & 7.87 & 34.55 & 25.23 & 7.97 & 0.61 & 7.36 \\
\hline LGG-574 & 36.67 & 67.00 & 42.98 & 2.00 & 9.16 & 3.68 & 11.26 & 4.27 & 11.99 & 43.68 & 25.50 & 8.13 & 0.66 & 7.47 \\
\hline LGG-460 & 34.33 & 65 & 49.46 & 2.40 & 8.18 & 3.67 & 10.51 & 3.92 & 10.70 & 36.27 & 25.67 & 8.34 & 0.75 & 7.59 \\
\hline LGG-407 & 38.00 & 70.00 & 56.18 & 3.80 & 8.75 & 3.20 & 10.60 & 5.85 & 11.37 & 38.88 & 25.98 & 8.75 & 0.68 & 8.07 \\
\hline Mean of lines & 37.00 & 68.13 & 44.61 & 2.24 & 6.83 & 3.29 & 10.59 & 4.29 & 10.33 & 38.44 & 25.30 & 8.17 & 0.67 & 7.38 \\
\hline \multicolumn{15}{|l|}{ Testers } \\
\hline Pusa Vishal & 37.67 & 68.00 & 40 & 1.06 & 4.33 & 3.04 & 9.04 & 4.33 & 9.86 & 39.90 & 24.26 & 7.49 & 0.61 & 6.88 \\
\hline PM-5 & 38.33 & 69.33 & 42.7 & 1.90 & 4.02 & 3.12 & 9.65 & 4.08 & 9.25 & 38.75 & 25.75 & 8.56 & 0.73 & 7.83 \\
\hline IPM-2-14 & 37.67 & 68.67 & 40.96 & 2.10 & 4.03 & 3.25 & 10.08 & 3.72 & 9.52 & 36.96 & 26.10 & 8.08 & 0.67 & 7.89 \\
\hline PM-110 & 38.00 & 70.33 & 40.14 & 1.72 & 3.99 & 2.97 & 10.08 & 4.43 & 8.13 & 36.48 & 24.51 & 7.94 & 0.66 & 7.21 \\
\hline Mean of testers & 37.91 & 69.08 & 40.38 & 1.69 & 4.09 & 3.09 & 9.71 & 4.13 & 9.44 & 37.40 & 25.15 & 8.07 & 0.66 & 7.35 \\
\hline \multicolumn{15}{|l|}{ Crosses } \\
\hline TM-96-2 xPusa Vishal & 39.33 & 71.33 & 48.81 & 1.93 & 4.50 & 3.89 & 10.64 & 4.03 & 10.51 & 33.19 & 25.26 & 6.59 & 0.63 & 5.96 \\
\hline TM-96-2 × PM-5 & 38.67 & 69.67 & 44.47 & 2.27 & 4.54 & 3.69 & 10.65 & 3.97 & 14.94 & 39.99 & 25.29 & 6.93 & 0.78 & 6.15 \\
\hline TM-96-2 × IPM-2-14 & 38.33 & 69.67 & 41.18 & 1.67 & 9.56 & 4.28 & 10.10 & 4.25 & 14.09 & 44.97 & 23.55 & 7.01 & 0.65 & 6.36 \\
\hline TM-96-2 x PM-110 & 39.33 & 68.67 & 50.60 & 2.87 & 10.74 & 3.72 & 11.17 & 3.83 & 12.05 & 29.79 & 23.09 & 7.93 & 0.73 & 7.20 \\
\hline
\end{tabular}




\begin{tabular}{|c|c|c|c|c|c|c|c|c|c|c|c|c|c|c|}
\hline & & & & & & & & & & & & \multicolumn{3}{|c|}{ Cont... } \\
\hline Genotype & $\begin{array}{c}\text { Days to } \\
50 \% \\
\text { flowering }\end{array}$ & $\begin{array}{c}\text { Days to } \\
\text { maturity }\end{array}$ & $\begin{array}{l}\text { Plant } \\
\text { height } \\
(\mathrm{cm})\end{array}$ & $\begin{array}{c}\text { Number } \\
\text { of } \\
\text { branches }^{-1} \\
\text { plant }^{-1}\end{array}$ & $\begin{array}{c}\text { Number } \\
\text { of } \\
\text { clusters }^{-1} \\
\text { plant }^{-1}\end{array}$ & $\begin{array}{l}\text { Number } \\
\text { of pods } \\
\text { cluster }^{-1}\end{array}$ & $\begin{array}{c}\text { Number } \\
\text { of seeds } \\
\text { pod }^{-1}\end{array}$ & $\begin{array}{c}100 \\
\text { seed } \\
\text { weight } \\
(\mathrm{g})\end{array}$ & $\begin{array}{l}\text { Seed } \\
\text { yield } \\
\text { plant }^{-1} \\
(\mathrm{~g})\end{array}$ & $\begin{array}{c}\text { Harvest } \\
\text { index } \\
(\%)\end{array}$ & $\begin{array}{c}\text { Total } \\
\text { protein } \\
\text { content } \\
(\%)\end{array}$ & $\begin{array}{c}\text { Total } \\
\text { sugars } \\
(\%)\end{array}$ & $\begin{array}{l}\text { Reducing } \\
\text { sugars } \\
(\%)\end{array}$ & $\begin{array}{l}\text { Non- } \\
\text { reducing } \\
\text { sugars } \\
(\%)\end{array}$ \\
\hline MGG-295 $\times$ PM-5 & 39.67 & 67.67 & 47.91 & 1.86 & 7.94 & 3.58 & 11.15 & 3.53 & 9.84 & 39.31 & 24.06 & 7.98 & 0.52 & 7.46 \\
\hline MGG-295 × IPM-2-14 & 38.67 & 70.67 & 42.87 & 1.17 & 8.24 & 3.37 & 11.45 & 5.03 & 12.65 & 39.40 & 22.39 & 6.89 & 0.69 & 6.20 \\
\hline MGG-295 × PM-110 & 38.67 & 69.67 & 38.80 & 1.23 & 3.80 & 3.26 & 11.67 & 5.57 & 8.82 & 41.37 & 23.59 & 7.04 & 0.75 & 6.29 \\
\hline LGG-574 $\times$ Pusa Vishal & 35.00 & 66.33 & 47.75 & 2.65 & 10.98 & 4.15 & 12.00 & 3.86 & 16.17 & 45.46 & 24.89 & 7.85 & 0.71 & 7.13 \\
\hline LGG-574 × PM-5 & 35.67 & 68.67 & 46.77 & 3.11 & 12.05 & 3.73 & 10.79 & 3.66 & 15.79 & 44.74 & 24.27 & 7.76 & 0.73 & 7.04 \\
\hline LGG-574 × IPM-2-14 & 38.33 & 68.00 & 37.83 & 1.13 & 5.83 & 3.69 & 10.66 & 4.55 & 8.35 & 42.23 & 22.40 & 7.96 & 0.53 & 7.42 \\
\hline LGG-574 × PM-110 & 38.33 & 67.00 & 38.47 & 1.15 & 6.79 & 3.42 & 10.16 & 5.49 & 10.14 & 41.12 & 22.59 & 7.17 & 0.59 & 6.58 \\
\hline LGG-460 $\times$ Pusa Vishal & 37.67 & 69.67 & 51.12 & 3.16 & 10.15 & 3.77 & 11.98 & 4.05 & 15.28 & 43.64 & 25.21 & 8.29 & 0.74 & 7.55 \\
\hline LGG-460× PM-5 & 38.67 & 69.67 & 43.77 & 1.66 & 8.99 & 3.69 & 10.77 & 3.78 & 11.44 & 28.95 & 22.64 & 7.52 & 0.65 & 6.87 \\
\hline LGG-460 ×IPM-2-14 & 40.67 & 71.00 & 49.87 & 2.75 & 11.23 & 4.37 & 11.44 & 3.36 & 15.07 & 43.14 & 25.33 & 8.50 & 0.76 & 7.74 \\
\hline LGG-407 ×Pusa Vishal & 40.67 & 69.00 & 41.59 & 1.36 & 8.75 & 4.12 & 10.77 & 3.83 & 11.17 & 42.91 & 25.08 & 7.35 & 0.61 & 6.73 \\
\hline LGG-407 ×PM-5 & 35.10 & 64.33 & 51.22 & 2.50 & 11.07 & 3.83 & 11.04 & 3.72 & 14.11 & 42.77 & 25.36 & 9.50 & 0.81 & 8.69 \\
\hline LGG-407 xIPM-2-14 & 41.67 & 68.67 & 46.62 & 2.06 & 11.56 & 3.47 & 10.98 & 3.44 & 13.05 & 39.57 & 22.24 & 7.46 & 0.65 & 6.81 \\
\hline LGG-407 × PM-110 & 40.00 & 68.67 & 44.70 & 1.66 & 7.14 & 3.58 & 9.66 & 3.86 & 8.45 & 38.55 & 24.18 & 7.23 & 0.55 & 6.69 \\
\hline Mean of crosses & 38.60 & 68.78 & 45.36 & 1.99 & 8.83 & 3.77 & 10.90 & 4.05 & 12.45 & 40.09 & 23.82 & 7.48 & 0.66 & 6.82 \\
\hline General mean & 38.23 & 68.71 & 44.54 & 1.99 & 7.83 & 3.59 & 10.69 & 4.10 & 11.67 & 39.44 & 24.26 & 7.67 & 0.67 & 6.99 \\
\hline S.E. & 1.80 & 1.77 & 1.67 & 0.12 & 0.34 & 0.18 & 0.43 & 0.21 & 0.78 & 2.21 & 0.33 & 0.15 & 0.02 & 0.17 \\
\hline C.V. & 5.79 & 3.16 & 4.58 & 7.96 & 5.44 & 6.42 & 5.02 & 6.40 & 13.18 & 6.89 & 1.67 & 2.39 & 2.97 & 3.04 \\
\hline
\end{tabular}




\section{Int.J.Curr.Microbiol.App.Sci (2018) 7(11): 1771-1779}

Table.2 Analysis of variance for combining ability for different quantitative characters in greengram

\begin{tabular}{|c|c|c|c|c|c|c|c|c|c|c|c|c|c|c|c|}
\hline Source & df. & $\begin{array}{c}\text { Days to } \\
50 \% \\
\text { flowering }\end{array}$ & $\begin{array}{l}\text { Days to } \\
\text { maturity }\end{array}$ & $\begin{array}{c}\text { Plant } \\
\text { height } \\
(\mathrm{cm})\end{array}$ & $\begin{array}{c}\text { Numberof } \\
\text { branches } \\
\text { plant }^{-1}\end{array}$ & $\begin{array}{c}\text { Numberof } \\
\text { clusters } \\
\text { plant }^{-1}\end{array}$ & $\begin{array}{l}\text { Number } \\
\text { of pods } \\
\text { cluster }^{-1}\end{array}$ & $\begin{array}{l}\text { Numberof } \\
\text { seeds pod } \\
\quad 1\end{array}$ & $\begin{array}{l}100 \text { seed } \\
\text { weight } \\
\text { (g) }\end{array}$ & $\begin{array}{l}\text { Seed } \\
\text { yield } \\
\text { plant }^{-1} \\
(\mathrm{~g})\end{array}$ & $\begin{array}{c}\text { Harvest } \\
\text { index } \\
(\%)\end{array}$ & $\begin{array}{c}\text { Total } \\
\text { protein } \\
\text { content } \\
(\%)\end{array}$ & $\begin{array}{l}\text { Total } \\
\text { sugars } \\
(\%)\end{array}$ & $\begin{array}{l}\text { Reducingsugars } \\
(\%)\end{array}$ & $\begin{array}{c}\text { Non- } \\
\text { reducingsugars } \\
(\%)\end{array}$ \\
\hline Replications & 2 & 4.63 & 1.53 & 8.30 & 0.08 & 0.63 & 0.10 & 0.09 & 0.21 & 1.70 & 4.10 & 0.11 & 0.13 & 0.01 & 0.18 \\
\hline Treatments & 28 & $9.12 *$ & $8.85^{*}$ & $70.94 * *$ & $1.47 * *$ & $25.64 * *$ & $0.45 * *$ & $1.43 * *$ & $1.24 * *$ & $18.91 * *$ & $52.30 * *$ & $4.83 * *$ & $1.69 * *$ & $0.02 * *$ & $1.64 * *$ \\
\hline Parents & 8 & $6.73 * *$ & $11.42 *$ & $96.61 * *$ & $2.02 * *$ & $16.29 * *$ & $0.24 * *$ & $1.18 * *$ & $1.36 * *$ & $5.61 *$ & $21.83^{*}$ & $1.69 * *$ & $0.47 * *$ & $0.01 * *$ & $0.49 * *$ \\
\hline Crosses & 19 & $9.21 *$ & 8.19 & $57.13 * *$ & $1.36 * *$ & $20.80 * *$ & $0.26 * *$ & $1.12 * *$ & $1.22 * *$ & $18.81 * *$ & $63.52 * *$ & $4.44 * *$ & $1.90 * *$ & $0.02 * *$ & $1.77 * *$ \\
\hline Lines & 4 & $11.83^{* *}$ & $19.10 * *$ & $150.69 * *$ & $3.08 *$ & $20.01 * *$ & $0.39 * *$ & 0.52 & $2.45 * *$ & $7.80 *$ & $35.45 * *$ & $1.49 * *$ & $0.47 * *$ & $0.01 * *$ & $0.44 * *$ \\
\hline Testers & 3 & 0.31 & $2.97 *$ & $17.06 * *$ & 0.61 & 0.08 & $0.04 *$ & $0.72 * *$ & $0.30 * *$ & $1.68 *$ & $8.57 * *$ & $2.45^{* *}$ & $0.57 * *$ & $0.01 * *$ & $0.71 * *$ \\
\hline $\begin{array}{l}\text { Lines } x \\
\text { Testers }\end{array}$ & 12 & 7.47 & 7.48 & $58.84 * *$ & $1.36 * *$ & $24.87 * *$ & $0.20 * *$ & $1.34 * *$ & $1.34 * *$ & $19.01 * *$ & $65.33 * *$ & $3.45^{* *}$ & $1.50 * *$ & $0.02 * *$ & $1.62 * *$ \\
\hline $\begin{array}{l}\text { Parents vs } \\
\text { Crosses }\end{array}$ & 1 & $26.48 * *$ & 0.97 & $128.20 * *$ & 0.02 & $192.32 * *$ & $5.76 * *$ & $9.17 * *$ & $0.56 * *$ & $127.03 * *$ & $82.85 * *$ & $37.27 * *$ & $7.32 * *$ & 0.01 & $8.21 * *$ \\
\hline Error & 56 & 4.91 & 4.73 & 4.24 & 0.03 & 0.18 & 0.05 & 0.29 & 0.07 & 2.20 & 7.39 & 0.17 & 0.03 & 0.01 & 0.05 \\
\hline $\begin{array}{l}g c a \\
\text { variance }\end{array}$ & & 0.3500 & 0.1010 & 0.0750 & 0.0120 & 0.9050 & 0.0120 & 0.0460 & 0.0290 & 0.0120 & 0.4730 & 0.2500 & 0.0910 & 0.0020 & 0.0260 \\
\hline sca variance & & 0.7580 & 1.3930 & 18.1500 & 0.4480 & 8.2400 & 0.0480 & 0.3610 & 0.4320 & 5.5460 & 19.0390 & 1.1400 & 0.4930 & 0.0080 & 0.5300 \\
\hline GCA/SCA & & 0.4617 & 0.0725 & 0.0041 & 0.0268 & 0.1098 & 0.2500 & 0.1274 & 0.0671 & 0.0022 & 0.0248 & 0.2193 & 0.1846 & 0.2500 & 0.0491 \\
\hline \multicolumn{16}{|c|}{$\begin{array}{l}\text { Significant at } 5 \% \text { level } \\
\text { Significant at } 1 \% \text { level }\end{array}$} \\
\hline
\end{tabular}




\section{Int.J.Curr.Microbiol.App.Sci (2018) 7(11): 1771-1779}

Table.3 Estimates of general combining ability ( $\mathrm{gca}$ ) effects of parents for seed yield, yield components and nutritional quality traits in greengram

\begin{tabular}{|c|c|c|c|c|c|c|c|c|c|c|c|c|c|c|}
\hline Genotype & $\begin{array}{c}\text { Days to } \\
50 \% \\
\text { flowering }\end{array}$ & $\begin{array}{l}\text { Days to } \\
\text { maturity }\end{array}$ & $\begin{array}{c}\text { Plant } \\
\text { height } \\
(\mathrm{cm})\end{array}$ & $\begin{array}{c}\text { Number } \\
\text { of } \\
\text { branches } \\
\text { plant }^{-1}\end{array}$ & $\begin{array}{c}\text { Number } \\
\text { of } \\
\text { clusters }^{-1} \\
\text { plant }^{-1}\end{array}$ & $\begin{array}{l}\text { Number } \\
\text { of pods } \\
\text { cluster }^{-1}\end{array}$ & $\begin{array}{c}\text { Number } \\
\text { of seeds } \\
\text { pod }^{-1}\end{array}$ & $\begin{array}{c}100 \text { seed } \\
\text { weight } \\
(\mathrm{g})\end{array}$ & $\begin{array}{l}\text { Seed } \\
\text { yield } \\
\text { plant }^{-1} \\
(\mathrm{~g})\end{array}$ & $\begin{array}{c}\text { Harvest } \\
\text { index } \\
(\%)\end{array}$ & $\begin{array}{c}\text { Total } \\
\text { protein } \\
\text { content } \\
(\%)\end{array}$ & $\begin{array}{c}\text { Total } \\
\text { sugars } \\
(\%)\end{array}$ & $\begin{array}{c}\text { Reducing } \\
\text { sugars } \\
(\%)\end{array}$ & $\begin{array}{c}\text { Non- } \\
\text { reducing } \\
\text { sugars } \\
(\%)\end{array}$ \\
\hline \multicolumn{15}{|l|}{ Lines } \\
\hline TM-96-2 & 0.89 & 1.04 & 0.92 & $0.20 * *$ & $-1.49 * *$ & 0.01 & -0.56 & $-0.18 * *$ & 0.18 & $-3.11 * *$ & $0.48 * *$ & 0.03 & $-0.03 * *$ & $-0.40 * *$ \\
\hline $\begin{array}{l}\text { MGG- } \\
295\end{array}$ & 0.96 & $1.13 * *$ & -0.43 & $-0.50 * *$ & $-1.09 * *$ & $-0.28 * *$ & 0.12 & $0.44 * *$ & $-1.40 * *$ & 0.28 & $-0.54 * *$ & $-0.59 * *$ & 0.01 & $-0.55 * *$ \\
\hline LGG-460 & -0.48 & -0.53 & $1.50 *$ & 0.18 & $1.69 * *$ & $0.19 *$ & $0.33 *$ & $-0.26 * *$ & $0.74^{*}$ & -1.32 & $0.12 *$ & -0.08 & $0.05 * *$ & $0.30 * *$ \\
\hline LGG-407 & $-0.73 *$ & -1.12 & 0.67 & $0.09 *$ & $0.81 * *$ & -0.02 & $0.29 * *$ & $-0.30 * *$ & $0.05^{*}$ & 0.86 & $0.35^{* *}$ & $0.33 * *$ & $0.01 * *$ & $0.42 * *$ \\
\hline $\mathbf{S E}\left(\mathbf{g}_{\mathbf{i}}\right)$ & 0.65 & 0.52 & 0.60 & 0.03 & 0.11 & 0.07 & 0.14 & 0.06 & 0.44 & 0.82 & 0.05 & 0.04 & 0.01 & 0.05 \\
\hline \multicolumn{15}{|l|}{ Testers } \\
\hline PM-5 & $-1.07 * *$ & -0.38 & $1.47 * *$ & $0.29 * *$ & 0.08 & $0.06^{*}$ & -0.02 & $-0.24 * *$ & $0.68 *$ & -0.94 & $0.47 * *$ & $0.47 * *$ & $0.03 * *$ & $0.43 * *$ \\
\hline IPM-2-14 & 1.04 & 0.82 & $-1.69 * *$ & $-0.23 * *$ & $0.46^{* *}$ & $0.07 *$ & 0.02 & 0.02 & $0.21 *$ & $1.77 *$ & $0.24 * *$ & $0.29 * *$ & $0.03 * *$ & 0.09 \\
\hline PM-110 & 0.67 & -0.45 & $-2.30 * *$ & $-0.23 * *$ & $-0.79 * *$ & $-0.17 * *$ & -0.22 & 0.02 & $-2.95 * *$ & $-2.06 * *$ & $-1.94 * *$ & $-0.17 * *$ & $-0.02 * *$ & $-0.21 * *$ \\
\hline $\mathbf{S E}\left(\mathbf{g}_{\mathbf{j}}\right)$ & 0.58 & 0.46 & 0.54 & 0.03 & 0.10 & 0.06 & 0.13 & 0.05 & 0.39 & 0.74 & 0.04 & 0.03 & 0.01 & 0.05 \\
\hline
\end{tabular}


Table.4 Estimates of specific combining ability ( $s c a$ ) effects of crosses for yield, yield components and nutritional quality traits in greengram

\begin{tabular}{|c|c|c|c|c|c|c|c|c|c|c|c|c|c|c|}
\hline Cross combinations & $\begin{array}{c}\text { Days to } \\
50 \% \\
\text { flowering }\end{array}$ & $\begin{array}{l}\text { Days to } \\
\text { maturity }\end{array}$ & $\begin{array}{c}\text { Plant } \\
\text { height } \\
(\mathrm{cm})\end{array}$ & $\begin{array}{c}\text { Number } \\
\text { of } \\
\text { branches } \\
\text { plant }^{-1}\end{array}$ & $\begin{array}{c}\text { Number } \\
\text { of } \\
\text { clusters }^{-1} \\
\text { plant }^{-1}\end{array}$ & $\begin{array}{l}\text { Number } \\
\text { of pods } \\
\text { cluster }^{-1}\end{array}$ & $\begin{array}{l}\text { Number } \\
\text { of seeds } \\
\text { pod }^{-1}\end{array}$ & $\begin{array}{c}100 \\
\text { seed } \\
\text { weight } \\
(\mathrm{g})\end{array}$ & $\begin{array}{c}\text { Seed } \\
\text { yield } \\
\text { plant }^{-1} \\
(\mathrm{~g})\end{array}$ & $\begin{array}{l}\text { Harvest } \\
\text { index } \\
(\%)\end{array}$ & $\begin{array}{c}\text { Total } \\
\text { protein } \\
\text { content } \\
(\%)\end{array}$ & $\begin{array}{l}\text { Total } \\
\text { sugars } \\
(\%)\end{array}$ & $\begin{array}{l}\text { Reducing } \\
\text { sugars } \\
(\%)\end{array}$ & $\begin{array}{c}\text { Non- } \\
\text { reducing } \\
\text { sugars } \\
(\%)\end{array}$ \\
\hline TM-96-2 ×Pusa Vishal & 0.82 & $1.48^{*}$ & 0.02 & $-0.42 * *$ & $-3.08 * *$ & -0.18 & -0.22 & -0.03 & $-3.25^{* *}$ & $-5.02 * *$ & -0.04 & $-0.63 * *$ & $-0.03 *$ & -0.14 \\
\hline TM-96-2 × PM-5 & 0.82 & 0.22 & $-3.26 * *$ & $-0.21 * *$ & $-2.89 * *$ & -0.14 & 0.03 & $-0.27 *$ & 1.36 & $3.95^{*}$ & $0.52 * *$ & $0.25^{* *}$ & $0.05^{* *}$ & $-0.69 * *$ \\
\hline TM-96-2 × IPM-2-14 & -1.52 & -0.98 & $-3.40 * *$ & $-0.29 * *$ & $1.77 * *$ & $0.31^{*}$ & -0.56 & $0.36^{* *}$ & 0.99 & $6.21 * *$ & -0.02 & $-0.21^{*}$ & $-0.08^{* *}$ & -0.15 \\
\hline TM-96-2 × PM-110 & -0.12 & -0.72 & $6.64 * *$ & $0.92 * *$ & $4.20 * *$ & 0.00 & $0.75^{*}$ & -0.05 & 0.91 & $-5.14 * *$ & $-0.46^{* *}$ & $0.60 * *$ & $0.06^{* *}$ & $0.98^{* *}$ \\
\hline MGG-295 $\times$ Pusa Vishal & -0.10 & $-0.27 *$ & $2.68^{*}$ & 0.02 & $2.99 * *$ & 0.08 & $-1.11 * *$ & $-0.85^{* *}$ & 0.95 & -0.22 & $-1.19 * *$ & $-0.84 * *$ & 0.01 & $-0.83 * *$ \\
\hline MGG-295 × PM-5 & 1.90 & 0.13 & 1.52 & 0.08 & 0.11 & 0.15 & 0.05 & $-0.73 * *$ & $-1.89 *$ & -0.11 & $0.31 * *$ & $0.21^{*}$ & $-0.18^{* *}$ & $0.76^{* *}$ \\
\hline MGG-295 × IPM-2-14 & -1.10 & -0.07 & -0.38 & -0.08 & 0.04 & -0.18 & 0.30 & $0.52 * *$ & 1.41 & -2.73 & -0.17 & $0.29 * *$ & $0.06^{* *}$ & -0.16 \\
\hline MGG-295 × PM-110 & -0.70 & 0.20 & $-3.82 * *$ & -0.02 & $-3.15^{* *}$ & -0.05 & $0.76^{*}$ & $1.06 * *$ & -0.47 & 3.07 & $1.05^{* *}$ & $0.33 * *$ & $0.11 * *$ & 0.23 \\
\hline LGG-574 × Pusa Vishal & $-1.43 * *$ & -1.93 & $2.52^{*}$ & $0.47 * *$ & $1.82^{* * *}$ & $0.24 * *$ & $0.88 * *$ & $0.94 * *$ & $2.82 * *$ & $0.85^{*}$ & $0.68 * *$ & $0.48 * *$ & 0.06 & $0.40^{*}$ \\
\hline LGG-574 × PM-5 & -0.10 & $0.80^{*}$ & $2.59 *$ & $0.80 * *$ & $3.05^{* *}$ & $0.04 *$ & -0.09 & $0.43 * *$ & $2.11 *$ & $2.29 * *$ & 0.15 & $0.77 * *$ & 0.00 & $0.43 * *$ \\
\hline LGG-574 × IPM-2-14 & 0.57 & $1.93 *$ & $-3.19^{*}$ & $-0.65^{* *}$ & $-3.54 * *$ & -0.13 & -0.27 & $-0.71 * *$ & $-4.34 * *$ & -2.93 & $-0.52 * *$ & $0.46^{* *}$ & 0.01 & $0.28^{*}$ \\
\hline LGG-574 × PM-110 & 0.97 & $-0.80^{*}$ & -1.93 & $-0.63 * *$ & $-1.34 * *$ & -0.15 & -0.52 & $-0.65 * *$ & -0.59 & -0.21 & $0.31 * *$ & -0.16 & $-0.07 * *$ & $-0.25 *$ \\
\hline LGG-460 $\times$ Pusa Vishal & $-1.02 * *$ & -0.60 & 1.73 & $0.64 * *$ & $0.61 * *$ & $0.30^{*}$ & 0.52 & 0.06 & $0.87 * *$ & $3.65^{*}$ & $0.65^{* *}$ & $1.18^{*}$ & $-0.04 * *$ & $0.75^{* *}$ \\
\hline LGG-460× PM-5 & 0.65 & 1.80 & $-4.56^{* *}$ & $-0.99 * *$ & $-1.62 * *$ & -0.20 & -0.44 & 0.22 & $-3.31 * *$ & $-8.88 * *$ & $-1.54 * *$ & $-0.63 * *$ & 0.01 & $-0.67 * *$ \\
\hline LGG-460 × IPM-2-14 & 0.65 & $-1.07 *$ & $4.69 * *$ & $0.62 * *$ & 0.25 & $0.35^{*}$ & 0.18 & 0.16 & $0.80^{*}$ & 2.60 & $1.91 * *$ & $0.18^{*}$ & $0.05 * *$ & $0.54 * *$ \\
\hline LGG-460 $\times$ PM-110 & -0.28 & -0.13 & -1.86 & $-0.27 * *$ & $1.98 * *$ & 0.20 & -0.26 & $-0.45^{* *}$ & 1.65 & 2.63 & $-1.03 * *$ & $-0.36^{* *}$ & -0.01 & $-0.62 * *$ \\
\hline LGG-407 × Pusa Vishal & $1.73^{* * *}$ & 1.32 & $-6.97 * *$ & $-0.71 * *$ & $-1.12 * *$ & 0.21 & -0.06 & -0.12 & -1.39 & 0.74 & -0.10 & $-0.18^{*}$ & 0.01 & -0.18 \\
\hline LGG-407 × PM-5 & -3.27 & -2.95 & $3.72^{*}$ & 0.31 & $1.35^{* *}$ & 0.14 & 0.45 & $0.35^{* *}$ & $1.74 *$ & 2.76 & $0.56^{* *}$ & $0.94 * *$ & $0.12 * *$ & $1.03^{* *}$ \\
\hline LGG-407 ×IPM-2-14 & 1.40 & 0.18 & 2.28 & $0.40 * *$ & $1.47 * *$ & $-0.39 *$ & 0.34 & $-0.33^{*}$ & 1.15 & -3.15 & $-1.21 * *$ & $-0.36^{* *}$ & $-0.04 * *$ & $-0.51 * *$ \\
\hline LGG-407 × PM-110 & 0.13 & 1.45 & 0.97 & 0.01 & $-1.70 * *$ & 0.01 & $-0.73^{*}$ & 0.10 & -1.49 & -0.35 & $0.75^{* *}$ & $-0.41 * *$ & $-0.08 * *$ & $-0.34 * *$ \\
\hline $\mathbf{S E}\left(\mathbf{S}_{\mathrm{ij}}\right)$ & 1.31 & 1.04 & 1.20 & 0.07 & 0.22 & 0.14 & 0.29 & 0.12 & 0.89 & 1.65 & 0.10 & 0.08 & 0.01 & 0.11 \\
\hline
\end{tabular}

* $\quad$ Significant at $5 \%$ level

** $\quad$ Significant at $1 \%$ level 
Pusa Vishal was good parent for days to $50 \%$ flowering, number of clusters per plant, seed yield per plant and harvest index and IPM-214 for number of pods per cluster, total protein content and total sugars. LGG-460 serves as good parent for number of pods per cluster and seed yield per plant. These parents serve as potential reservoir of genes for their respective traits. Therefore these parents could be exploited in multiple crossing programme to synthesize a dynamic population with accumulation of most the favourable genes (Griffing, 1956).

The estimate of sca effects reveals the usefulness of a particular cross for exploitation of heterosis. The sca effects of twenty crosses evaluated in the present study were presented in table 4 . It was interesting to note that none of the crosses recorded significant sca effects in desirable direction for all the traits. The sca effects signify the role of non-additive gene effects mainly dominance gene effects (Nadarajan and Gunasekaran, 2005).

Among the crosses, LGG-574 x Pusa Vishal was identified as best specific combiner for days to $50 \%$ flowering, plant height, number of branches per plant, number of clusters per plant, number of pods per cluster, number of seeds per pod, hundred seed weight, seed yield per plant, harvest index, total protein content and total sugars. LGG-574 x PM-5 was found to be the next best cross with significant sca effects for days to maturity, plant height, and number of branches per plant, number of clusters per plant, number of pods per cluster,100 seed weight, seed yield per plant, harvest index, total sugars and nonreducing sugars. The cross LGG-460 x Pusa Vishal recorded significant sca effects for days to $50 \%$ flowering, number of branches per plant, number of clusters per plant, number of pods per cluster, seed yield per plant, harvest index, total protein content and non-reducing sugars. LGG-460 x IPM-2-14 showed significant sca effects for days to maturity, plant height, number of branches per plant, number of pods per cluster, seed yield per plant, total protein content, reducing sugars and non-reducing sugars. LGG-407 x PM-5 exhibited significant sca effects for number of branches per plant, number of clusters per plant, 100 seed weight, seed yield per plant, total protein content, total sugars, reducing sugars and non-reducing sugars.

The present study was carried out for identification of best parents and superior crosses for yield, yield components and nutritional traits. An overall view of $\mathrm{gca}$ and sca effects revealed LGG-574, LGG-460, LGG-407, Pusa Vishal, PM-5 and IPM-2-14 as promising parents and the crosses, LGG574 x Pusa Vishal, LGG-574 x PM-5, LGG$460 \times$ Pusa Vishal, LGG-460 x IPM-2-14 and LGG-407 x PM-5 as promising hybrids for various yield, yield components and nutritional quality traits. Therefore, these crosses could be successfully employed in further breeding programmes so as to isolate desirable transgressive segregants for yield, yield components and nutritional quality traits. Further it was evident from the study that additive and non- additive gene action plays a significant role in the expression of most of yield components and nutritional quality traits. Therefore the superior segregants can be handled through biparental mating preceeding selection to harness the full benefits of both additive and non-additive gene action.

\section{References}

Allard, R.W. (1960). Principles of plant breeding. John Willey and Sons Inc. New York.

Griffing, B. 1956. Concept of general and specific combining ability in relation to 
diallel crossing systems. Australian J. Biol Sci., 9:463-93.

Kamleshwar, K., Yogendra, P., Mishra, S. B., Pandey, S. S. and Ravi, K. 2014. Study on genetic variability, correlation and path analysis with grain yield and yield attributing traits in green gram [Vigna radiata (L.) Wilczek]. The Bioscan. 8(4): 1551-1555.

Kempthorne, O. 1957. An introduction to genetic statistics. John Wiley and Sons, New York.

Nadarajan, N. and Gunasekaran, M. 2005. Quantitative Genetics and Biometrical Techniques in Plant Breeding. Kalyani Publ., New Delhi.
Panse, V.G. and Sukhatme, P.V. 1985. Statistical methods for Agricultural workers, Indian Council of Agricultural Research, New Delhi.

Reddy, K.R.D., Venkateswarlu, O., Obaiah, M.C and Jyothi, S.G.L. 2011a. Heterosis for yield and yield components in greengram (Vigna radiata (L) Wilczek). Legume Research. 34(3): 207-211.

Singh, N.B. and Harisingh. 1985. Heterosis and combining ability for kernel size in Rice. Indian J. Genetics and Plant Breeding, 45(2): 181-185.

\section{How to cite this article:}

Kalpana, S., N.V. Naidu and Reddy, D.M. 2018. Combining Ability Studies for Yield, Yield Components and Nutritional Traits in Greengram (Vigna radiata (L.) Wilczek). Int.J.Curr.Microbiol.App.Sci. 7(11): 1771-1779. doi: https://doi.org/10.20546/ijcmas.2018.711.202 